Präv Gesundheitsf 2019 · 14:349-354

https://doi.org/10.1007/s11553-019-00715-6

Eingegangen: 1. Januar 2019

Angenommen: 11. März 2019

Online publiziert: 2. April 2019

(c) Der/die Autor(en) 2019

Settingspezifische Gesundheitsförderung für ältere Menschen findet häufig in der Stadt oder dem Wohnbezirk statt - so auch im Projekt "Gesund älter werden in Wien". Gesundheitsförderung für Ältere ist ein kommunales Praxisfeld, jedoch gibt es kaum Studien, die die sozialräumliche Aktivität Älterer mit Gesundheitsförderung verknüpfen. Angesichts des demografischen Wandels, der Diversifizierung und Verdichtung städtischer Lebensweisen wird es zunehmend wichtiger, Aktivitätspotentiale Älterer in der Stadt differenziert und zielgruppenspezifisch zu erkunden.

\section{Hintergrund}

\section{Demografischer Wandel}

Knapp ein Viertel der Wiener ist über 60 Jahre alt; die Tendenz ist mit Blick aufden demografischen Wandel steigend. Daraus entstehen besondere Bedürfnisse, auf die die Stadt Wien - insbesondere die Gesundheitsförderung und die Stadtteilarbeit - in der Planung von Angeboten verstärkt Rücksicht nimmt.

Mobilität und Mobilitätsanlässe nehmen im Alter tendenziell ab, daher gewinnt die unmittelbare Wohnumgebung an Bedeutung [3]. In vielen Bezirksteilen Wiens ist auch im Alter Mobilität möglich. Dies ist v.a. durch ein gut ausgebautes und in weiten Teilen barriere-

Aus Gründen der besseren Lesbarkeit wird in diesem Beitrag überwiegend das generische Maskulinum verwendet. Dies impliziert immer beide Formen, schließt also die weibliche Form mit ein.

Katharina Resch (1D · Julia Demmer · Anna FassI

Universität Wien, Wien, Österreich

\title{
Sozialraum und Altern: Aktivitätspotentiale von Älteren in der Gesundheitsförderung
}

freies, öffentliches Verkehrsnetz möglich. Auch die Angebotslage für ältere Menschen (z. B. Bewegung, Kreatives, Geselliges) ist in Wien gut ausgebaut, insbesondere durch stadtteilbezogene Organisationen, Vereine und Bildungsträger sowie bestehende Einrichtungen für Ältere der Stadt Wien. Eine Herausforderung für bestehende Angebote ist es, in ihrer Adressierung und Gestaltung auf veränderte Alternsprozesse und differenzierte Zielgruppen zu reagieren.

\section{Sozialraum Wien}

Wien als Untersuchungsraum der Studie stellt modellhaft eine ,typische“ europäische Stadt dar, die von Dichte, Heterogenität und von einer urbanen Lebensweise gekennzeichnet ist. Großstädte implizieren eine starke soziale Mischung und Überschneidung von gemeinsam genutztem Sozialraum [11]. Raumeinheiten (z. B. U-Bahnhaltestellen, Fußgängerund Begegnungszonen) strukturieren die Großstadt und ermöglichen unterschiedliches Nutzungsverhalten.

Menschen, die schon lange in ihrem Stadtteil leben, nehmen diesen sozialräumlich intensiv wahr. Routinen prägen ihre Wege in der Stadt. Bekannte Routen, Orte und Aktivitäten ermöglichen es, mit einer subjektiv verfügbaren „kognitiven Landkarte" [14] sicher unterwegs zu sein und biografische Routinen im Ruhestand fortzusetzen. Alltagsroutinen im Stadtteil werden aus verschiedenen Gründen im Alter auch (neu) entwickelt: Veränderungen der Wohnlage (z. B. Umzug in eine barrierefreie Wohnung), körperliche Belastungen oder plötzliche Freizeit im Ruhestand.

\section{Das Projekt}

Das Projekt „Gesund älter werden in Wien" wird in 9 von 23 Wiener Gemeindebezirken umgesetzt [15], die als heterogen beschrieben werden können. Die Unterschiede zeigen sich deutlich bei der Betrachtung einzelner Kennzahlen, u. a. der Bevölkerungsdichte, der Bezirksfläche oder dem durchschnittlichen Jahresnettoeinkommen der Einwohner [12].

Das Projekt wird von der Wiener Gesundheitsförderung umgesetzt und aus den Mitteln des Wiener Landesgesundheitsförderungsfonds finanziert (Laufzeit: 2017-2020). Die primäre Zielgruppe des Projekts sind nicht mehr erwerbstätige Männer und Frauen zwischen 60 und 75 Jahren. „Gesund älter werden in Wien" besteht aus fünf Projektphasen. Der vorliegende Beitrag enthält die Studienergebnisse der ersten Projektphase, in der sozialräumliches Altern im Fokus steht. Parallel dazu findet die Kontaktphase statt, in der für ältere Bewohner in allen 9 Projektbezirken konsumfreie Treffen im Stadtteil („Grätzel-Cafés“) organisiert werden. Davon ausgehend werden partizipative Maßnahmen mit Älteren zur sozialen Teilhabe und Steigerung ihrer Gesundheitskompetenz umgesetzt. Die letzte Phase widmet sich der Sicherung der Nachhaltigkeit.

\section{Ziele und Fragestellung}

Die Studie legte eine sozialräumliche Perspektive auf das Altern in der Großstadt Wien. Die zentralen Forschungsfragen der Studie lauteten: (1) Welche Formen 
der sozialräumlichen Aneignung lassen sich bei älteren Bewohnern rekonstruieren - mit Fokus auf Gesundheit, Mobilität, Angebotsnutzung und soziale Eingebundenheit? (2) Welche Aktivitätspotentiale weisen ältere Wiener auf, und welche Aktivitätstypen können unterschieden werden?

Um Wissen über das Aktivitätsverhalten Älterer in Wien zu generieren, wurden in dieser Arbeit sozialräumliche und aneignungstheoretische Methoden und Theorien genutzt.

\section{Methodik}

Insgesamt zählen 19 Frauen und 11 Männer im Alter von 61 bis 84 Jahren zur Studienpopulation. Es fanden 27 Stadtteilbegehungen mit insgesamt 30 Bewohnern zwischen Mai und Juli 2018 statt und dauerten zwischen 1 und $3 \mathrm{~h}$ (drei Begehungen fanden als Paarbegehungen statt). Stadtteilbegehungen zählen in der Sozialforschung zu den "mobile methods" [2]. Diese werden oft mit anderen Erhebungsverfahren verknüpft (z. B. Interviews, visuelle Daten). „Mit einer kleinen Gruppe von Menschen wird der Stadtteil auf einer von ihnen eingeschlagenen Route begangen und zugleich werden ihre Interpretationen der sozialräumlichen Qualitäten dieser Räume mittels Diktiergerät und Fotoapparat dokumentiert" [9]. Die in den Stadtteilbegehungen zurückgelegten und dokumentierten Wege lassen sich in zwei Grundformen unterteilen: Alltagsrouten und Expertenrouten. Die Alltagsrouten (,trails“) führten entlang von Wegen, die von den älteren Personen im Alltag regelmäßig zurückgelegt werden, während die Expertenrouten (,tours") eigens für die Stadtteilbegehungen von den Bewohnern gewählt wurden [10]. Der zurückgelegte Weg wurde mittels App aufgezeichnet oder in Bezirksplänen rekonstruiert. Die Auswertung erfolgte durch minimale und maximale Kontrastierung in Anlehnung an Strauss/Corbin [13]. Abschließend folgte eine Generalisierung und Typenbildung nach Kluge [8] sowie die Rückkoppelung mit Literatur der Alterns-, Sozialraumund Gesundheitsforschung.

Zusätzlich wurde in allen 9 Projektbezirken je eine Fokusgruppendiskussion durchgeführt $(n=55)$. Alle Teilnehmenden haben einen Basisdatenleitfaden ausgefüllt, um sozialstatistische Daten wie Alter, Geschlecht, Wohndauer im Bezirk etc. anonymisiert zu erheben.

\section{Ergebnisse}

\section{Sozialraum und Altern}

Die Studie ermöglicht Einblicke in sozialräumliche Aneignungsprozesse von Bewohnern vor dem Hintergrund des gesunden Älterwerdens in der Stadt. Der Raum kann als konkretes, situatives Umfeld (z. B. Stadtteil) im Sinne eines sozialgeographisch umgrenzten Raums, aber auch als ein sozial konstruierter Raum ins Blickfeld rücken. Räume werden als „keine absoluten Einheiten, sondern ständig (re)produzierte Gewebe sozialer Praktiken " und Prozesse betrachtet [7]. Zugleich haben sich räumliche Ordnungen, welche sozial ungleiche Positionierungen fördern und reproduzieren, historisch entwickelt und manifestiert. Dies wirft Fragen auf, wie bestehende räumliche Ordnungen - mit Bewohnern gemeinsam - gesundheitsförderlich gestaltet werden können.

Das Konzept der sozialräumlichen Aneignung wurde bislang in der sozialpädagogischen Forschung zu Lebenswelten von Kindern und Jugendlichen genutzt $[4,6]$, aber auch in der Sozialarbeitsforschung mit Älteren aufgegriffen $[1,5]$. Wolter [14] nennt in ihrer Studie zu „Aneignung und Verlust des städtischen Raums im Alter" die wahrnehmungsbezogene Aneignung in drei Kategorien: (1) Wahrnehmen, (2) Handeln und (3) Gestalten. Diese Kategorien sind theoretischer Hintergrund für die Auswertung der Studienergebnisse und können folgendermaßen beschrieben werden:

(1) Menschen nehmen ihre räumliche Umwelt im Sinne einer räumlich-materiellen „Begrenzung“ wahr. Die Stadt besteht aus Wegen, Orten und Zugängen, die unterschiedliche Funktionen haben. Diese funktionalen und semantischen Bedeutungen werden vor dem Hintergrund des jeweiligen sozialen und kulturellen Kapitals wahrgenommen und gelten als variabel. Die subjekti- ve "Wahrnehmungskompetenz" kann von „Wahrnehmungsbeeinträchtigungen" beeinflusst werden (z.B. Seh- und Hörvermögen; [14]).

(2) Auf der Handlungsebene rücken Angebotsstrukturen in den Blick. Als besonders bedeutsam beschreibt Wolter [14] Angebote mit "Aufforderungscharakter", wie nutzungsoffene Räume und Treffpunkte, niederschwellige und konsumfreie Angebote (z. B. Nachbarschaftscafé, Märkte, Mittagstisch), die einen Möglichkeitsraum eröffnen. Solche Strukturen unterstützen das Spacing, d.h. dass Ältere sich Raum verschaffen. Wie Ältere ihre Handlungskompetenz einschätzen, begrenzt ihre räumliche Aneignung und ist von den jeweiligen Einstellungen und Normvorstellungen abhängig. Sozialräumliche Aneignungsprozesse älterer Menschen werden von lokalen Gegebenheiten des Sozialraums, der individuellen Lebenslage und gesellschaftlichen Altersbildern beeinflusst [14].

(3) Sozialräume können auch von Älteren (gesundheitsfördernd) mitgestaltet werden. Insbesondere ältere Menschen haben durch den Zugewinn an Zeitressourcen ein Aktivitätspotential, sich an partizipativen Prozessen zu beteiligen, „sie verfügen über mehr gestaltbare Zeit sowie Fachwissen und Lebenserfahrung" und wohnen häufig bereits länger an einem Ort, wodurch sie zu Experten für ihren Bezirk werden können [14].

\section{Wahrnehmungsebene}

In der Analyse der 27 Routen konnten folgende Themenfelder als relevante Wahrnehmungskategorien identifiziert werden: Lärmentwicklung, Grün- und Betonflächen, bauliche Veränderungen und wahrgenommene Bevölkerungsstrukturen. Ältere Menschen berichten beispielsweise von Lärm und Gerüchen und unterschiedlichen Formen der Lärmentwicklung (z. B. gleichbleibender Straßenlärm, laute Gespräche vor dem Haus). Viele suchen zum Ausgleich Ruhe- und Erholungsorte auf. Marianne (67 Jahre) findet dies in ihrem Schrebergarten: „Also, jede Zeit, die ich erübrigen kann, bin ich im Garten“. Gleichzeitig nimmt Marianne auch ihren eigenen in- 
nerstädtischen Wohnbezirk als begrünt wahr und erzählt: „Nein, wir haben doch eh Parks. Wenn ich das Grüne nicht suche, dann sehe ich nichts." Grün- und Betonflächen werden ebenso wahrgenommen wie Lärm: Eine Bewohnerin eines Außenbezirks Wiens antwortet während der Stadtteilbegehung auf die Frage, wie sie ihren Bezirk wahrnimmt: „Da wurde extra ein Auffangbecken für den Regen geschaffen, damit diese Neubauten da unten, da ist alles nur Beton. Das stört mich sehr. Ja der Beton ist leichter zu reinigen wie eine Wiese, aber wie gesagt, es ist auch da alles zubetoniert worden“ (Renate, 79 Jahre). Den Stadtteilbegehungen zufolge nehmen Ältere einerseits sehr ruhige und andererseits gesellige Orte aktiv wahr. So stellen Grünflächen, Märkte und Spielplätze Orte dar, die als gesundheitsbezogene Erholungsräume wahrgenommen werden. Doris (75 Jahre) hält sich gerne im Kongresspark auf, mit dem sie „Leben“, d. h. soziale Teilhabe und intergenerative Erlebnisse verbindet. Edith (74 Jahre) ist in ihrem Bezirk vorwiegend zu Fuß und auf Krücken unterwegs. Sie dreht ihre „Runden“, um rauszukommen, und besucht hierfür auch gerne belebte Plätze. Besonders gerne hat sie den Rochusplatz und -markt, denn dort beobachtet sie das Treiben der Menschen, der Verkäufer und Kinder. So zeigt sich die Koppelung von Sozialraum und sozialer Teilhabe, wenn diese auch nicht zu einer Interaktion führt, sondern die Frauen in der Rolle der Beobachterinnen verbleiben. Die Befragten erwähnen auch bauliche Veränderungen, da Wien eine schnell wachsende Stadt darstellt, die neue Bezirksteile hervorbringt. Städtebauliche Veränderungen oder Zuwanderung führen zu einer angepassten Wahrnehmung des eigenen Wohnumfelds. Edith (74 Jahre) wohnt seit ihrer Kindheit im Bezirk: „Und so und in der Nähe der Arenbergpark, der ja sehr schön ist, aber leider Gottes halt immer mehr in Ghettos sich verkleinert. (...) das Café ist eingezäunt, der Kinderspielplatz ist eingezäunt, der Hundeplatz ist eingezäunt, dann das Urban Gardening ist eingezäunt (...). Ich will einen Park haben, wo Jung und Alt beieinander sind." In Bezug auf Bevölkerungsstrukturen nehmen Ältere ein

Präv Gesundheitsf 2019 · 14:349-354 https://doi.org/10.1007/s11553-019-00715-6

(c) Der/die Autor(en) 2019

\section{K. Resch · J. Demmer · A. Fassl}

\section{Sozialraum und Altern: Aktivitätspotentiale von Älteren in der Gesundheitsförderung}

\section{Zusammenfassung}

Hintergrund. Settingspezifische Gesundheitsförderung für ältere Menschen findet häufig in der Stadt oder dem Wohnbezirk statt. Die Stadt Wien hat über 1,8 Mio. Einwohner und $22 \%$ der in Wien lebenden Menschen sind über 60 Jahre alt. Gesundheitsfördernde Maßnahmen sollen niederschwellig und zielgruppenspezifisch im urbanen Setting entwickelt werden. Die zunehmende Heterogenität des Alters sowie die Einbindung nicht-aktiver Älterer stellt die Gesundheitsförderung jedoch vor neue Herausforderungen. Oftmals wird nur ein Teil der Älteren adressiert und erreicht.

Ziel. Das Projekt "Gesund älter werden in Wien" wirft einen Blick auf sozialräumliche Fragen der Gesundheitsförderung im Alter. Ältere Menschen sind durch ihre Lebenslage auf unterschiedliche Weise (sozial)räumlich aktiv.

Ergebnisse. Die Ergebnisse aus 27 Stadtteilbegehungen und 30 Interviews zeigen deutliche Unterschiede der sozialräumlichen Aneignung und Nutzung der Stadt Wien durch ältere Bewohner auf drei Ebenen: Wahrnehmung, Handlung und Gestaltung. Die Angebotsentwicklung und -nutzung wird sozialräumlich (etwa in Kern- und Außenbezirken) unterschiedlich beschrieben. Ältere Bewohner können anhand von vier verschiedenen Aktivitätspotentialen typisiert werden: (1) Vollaktivität, (2) erweiterbare Aktivität, (3) Aktivität trotz Belastung und (4) Verlust der Aktivität. Jeder dieser Typen weist unterschiedliche Implikationen für die Zielgruppenerreichung in der Gesundheitsförderung und Stadtteilarbeit auf.

Schlussfolgerungen. Die Nutzung der Typologie kann die Gesundheitsförderung dabei unterstützen, ihre Zielgruppenadressierung zu verfeinern.

Schlüsselwörter

Kommunale Gesundheitsförderung . Stadtteilbegehungen - Alternsforschung · Sozialräumliche Aneignung · Gesundheitsförderung im Alter

\section{Spacing and ageing: activating older people in health promotion}

\section{Abstract}

Background. Health promotion for older people oftentimes takes place in the urban setting, close to where older people live. Of the 1.8 million inhabitants in the city of Vienna, 22\% are older than 60 years of age. Health-promoting services should be developed in a low-threshold manner and be adequate for different older target groups in an urban setting. The increasing heterogeneity of age and the inclusion of non-active older people, however, pose challenges to health-promoting services. Often only fractions of older people are addressed and reached.

Objective. The project "Gesund älter werden in Wien" focuses on health promotion as a topic of ageing taking into consideration aspects associated with spacing. Older people have a diverse range of mobility and activity behaviours in urban areas.

Results. Results of the 27 walk alongs and 30 interviews with older people in Vienna show differences in a person's spacing and ageing in place in three categories: perception of the area, activity and participation. Also, service development and use are described differently depending on the respective district (i. e. in inner and outer districts). Older adults can be typified by different types of activity: (1) full activity, (2) expandable activity, (3) activity despite handicap, (4) loss of activity. This again has implications for reaching target groups in health promotion and neighbourhood work.

Conclusion. The use of this typology can support health-promoting services to refine their strategies to address the target group of older people.

\section{Keywords}

Health promotion in urban areas - Mobile methods - Gerontology · Ageing in place . Health promotion with older people 
Tab. 1 Aktivitätstypologie älterer Menschen

\begin{tabular}{|c|c|c|}
\hline Aktivitätstypen & $\begin{array}{l}\text { Engagement } \\
\text { DIE AKTIVEN } \\
n=22\end{array}$ & $\begin{array}{l}\text { Disengagement } \\
\text { DIE BLOCKIERTEN } \\
n=5\end{array}$ \\
\hline $\begin{array}{l}\text { Dynamik } \\
\text { DIE FREISCHWEBENDEN } \\
n=12\end{array}$ & $\begin{array}{l}\text { Typ } 1 \\
\text { Erweiterbare Aktivität }\end{array}$ & $\begin{array}{l}\text { Typ } 3 \\
\text { Aktivität trotz Belastung }\end{array}$ \\
\hline $\begin{array}{l}\text { Statik } \\
\text { DIE VERORTETEN } \\
n=15\end{array}$ & $\begin{array}{l}\text { Typ } 2 \\
\text { Vollaktivität }\end{array}$ & $\begin{array}{l}\text { Typ } 4 \\
\text { Verlust der Aktivität }\end{array}$ \\
\hline
\end{tabular}

soziales Miteinander von Menschen verschiedener Altersgruppen wahr. Auch Zuwanderung oder "Überfremdung“ wird in bestimmten Bezirksteilen explizit erwähnt (etwa das Hören „fremder“ Sprachen). Zum einen beziehen sich Aussagen von Befragten auf Bezirksteile, in denen seit der Gastarbeiterbewegung ein zunehmender Anteil an Menschen mit Migrationsgeschichte lebt, zum anderen auf die Folgen der fluchtbedingten Migration der jüngeren Vergangenheit. Waltraud (73 Jahre): „Es ist irgendwie alles sehr fremd geworden (...). Der (...) Bezirk, manche Teile davon, wenn ich nicht dorthin muss und meistens muss ich nicht, meide ich sie wirklich. Es ist mir nicht angenehm [...]. Wien ist nicht nur eine liebenswerte, es ist auch eine der sichersten Großstädte der Welt und ich finde, das sollte man nicht dramatisieren." Zuwanderung wird als gesellschaftliche Veränderung auch sozialräumlich wahrgenommen. Für einen Teil der Befragten führt dies zu einer Anpassung im Bereich ihrer Mobilität.

\section{Handlungsebene}

Auf der Handlungsebene ging die Studie der Frage nach, wie sich Ältere in ihrem sozialräumlichen Aktivitätspotential unterscheiden. Während die kommunale Gesundheitsförderung und die Stadtteilarbeit meist den Auftrag haben, sozial benachteiligte Bewohner zu erreichen bzw. zu aktivieren, zeigen die Ergebnisse der Studie, dass sich nicht alle älteren Menschen aktivieren lassen und dass sich von bestehenden Angeboten nicht immer alle adressiert fühlen (wollen). Mithilfe einer Vierfeldertafel wurden vier Aktivitätstypen bestimmt (•Tab. 1). Diese vier empirischen Typen sind Idealtypen [8], sie verkörpern eine Vielzahl von typischen
Merkmalen der Gruppe (in der sozialen Realität können Mischtypen oder weitere Typen auftreten).

Die Aktivitätsachse beschreibt Idealtypen auf der Achse zwischen Engagement und Disengagement (in Anlehnung an Theorien der Alternsforschung), während die Mobilitätsachse diese auf der Achse zwischen Dynamik und Statik eingliedert (in Anlehnung an Theorien aus der Mobilitätsforschung).

\section{Typ 1: Erweiterbare Aktivität}

Ältere Menschen des Typs 1 gehen mehreren unterschiedlichen Aktivitäten in der Stadt nach und zählen damit zur Gruppe der Aktiven. Sie sind offen, neue Aktivitäten in ihr Repertoire aufzunehmen. Sie haben vielfältige Interessen und nutzen ein breites Angebot in der Stadt Wien. Im Vergleich zu Typ 2 sind sie nicht in einer bestimmten Organisation verankert (z. B. Organisation oder Verein im Stadtteil, Glaubensgemeinschaft) und engagieren sich nicht freiwillig. Sie verfügen über eine gute soziale Eingebundenheit (z. B. Familie, Freunde) und lernen auch neue Menschen kennen.

\section{Typ 2: Vollaktivität}

Zum Typus 2 zählen ältere Menschen, die als besonders aktivitätsorientiert und ressourcenstark beschrieben werden können. Diese sind mit ihren Tätigkeiten aktuell vollkommen ausgelastet. Viele in diesem Typus sind freiwillig tätig und seit vielen Jahren an eine intensive Aktivitätsausübung gewöhnt. Im Gegensatz zu Typ 1 sind sie eng an eine bestimmte Organisation oder Gruppe gebunden und dort sozial verankert (z. B. Pfarre, Verein). Die Gruppe des Typs 2 ist (im Gegensatz zu Typ 3) ressourcenstark. Ihre Haltung besteht darin, andere materiell, sozial, zeitlich, finanziell oder emotional zu unterstützen. Sie verfügen - wie auch Typ 1 - über eine sehr gute soziale Eingebundenheit. Sie haben aber derzeit keine Zeit oder kein Interesse, weitere Aktivitäten aufzunehmen (Vollaktivität).

\section{Typ 3: Aktivität trotz Belastung}

In dieser Gruppe befinden sich in unserem Sample ausschließlich alleinlebende ältere Menschen, die wenig sozial eingebunden und wenig familiär verortet sind. Zusätzlich sind sie gesundheitlich oder emotional belastet und aktuell weniger mobil als Typ 1 und 2, sodass sich ihr Aktivitätsradius insgesamt beschränkt. Die dem Typ 3 zugeordneten Bewohner gehen ihren Interessen nach, aber manchmal ist dies nicht wie gewünscht möglich, da Belastungen im Vordergrund stehen. Sie haben keine (Enkel-)Kinder oder wenig Kontakt zu Angehörigen. Im Gegensatz zu Typ 1 und 2 spiegelt sich in diesem Typus eine pessimistischere Lebenshaltung wider. Veränderungswünsche $z$. B. durch technische oder finanzielle Hilfen oder einen neuen Partner stehen im Vordergrund. Von ihrer Einschränkung werden sie daran gehindert, linear oder regelmäßig aktiv zu sein, und können dies nur fragmentiert oder sporadisch tun.

\section{Typ 4: Verlust der Aktivität}

Ältere Menschen des Typs 4 erleben einen Rückzug oder einen Verlust an Aktivität im Alter. Diese sind in der Familie verortet und ihre soziale Eingebundenheit dadurch bestimmt. Sie haben wenig Eigenantrieb oder Neugierde, etwas in der Stadt zu entdecken bzw. Angebote auszuprobieren, auch wenn diese räumlich naheliegen. Sie nehmen Angebote kaum wahr. Ihre Mobilität beschränkt sich gegenwärtig zumeist auf ihren Bezirk und die Wohnung ist ihr Lebensmittelpunkt. Es fällt ihnen eher schwer, Aktivitätsangebote zu besuchen, bei denen sie niemanden kennen. Aus diesem Grund und auch weil ihre familiäre Verortung als stark bezeichnet werden kann, findet ein Rückzug ins Private statt.

Die Ergebnisse zeigen, dass sich unterschiedliche Herausforderungen in der Zielgruppenerreichung Älterer in der Stadt ergeben, die für gesundheitsfördernde Projekte bedeutsam sind. 
- Menschen mit erweiterbarer Aktivität (Typ 1) sind am besten für gesundheitsfördernde Angebote zu erreichen, da sie auf der Suche nach passenden Aktivitäten sind.

- Ähnliches gilt für ältere Bewohner, die aufgrund ihres Aktivitätspotentials der Aktivität trotz Belastung (Typ 3) in ihrer Aktivitätsausübung blockiert sind: Diese können mit maßgeschneiderten Angeboten erreicht werden, wenn ihre Belastungen in der Angebotsstruktur Berücksichtigung finden.

- Ältere Menschen, die Typ 2 (Vollaktivität) und Typ 4 (Verlust der Aktivität) zugeordnet werden können, sind aus unterschiedlichen Gründen nur mit einem erhöhten Aufwand zu erreichen. Typ 4 ist nur im unmittelbaren Wohnumfeld oder in der Wohnung aufsuchend zu aktivieren, daher benötigen Institutionen für ihre Erreichung Ressourcen und zudem Argumentarien.

- Die Verorteten (Typ 2 Vollaktivität und 4 Verlust der Aktivität) können insgesamt schwieriger als die Freischwebenden für gesundheitsfördernde Maßnahmen aktiviert werden. Die Achse Dynamik-Statik ist demnach für die Zielgruppenerreichung relevanter.

Insgesamt konnten Bewohner, die in Bezug auf ihr Aktivierungspotential dem Typ 4 (Verlust der Aktivität) zugeordnet werden können und von sozialer Isolation betroffen sind, in den Stadtteilerhebungen kaum erreicht werden. Diese Menschen in der partizipativen Gesundheitsförderung $\mathrm{zu}$ erreichen, stellt eine bedeutende Herausforderung dar, die mit Hilfe der Typologie vorhersehbarer wird bzw. auch eng mit Fragen der Adressierung des Typs 4 zusammenhängt.

\section{Gestaltungsebene}

Die dritte Auswertungsebene bezieht sich auf die Ebene der Gestaltung des städtischen Raums im Alter. Die aktiven Älteren (Typ 1 und 2) sind bereits aktiv, wobei Personen des Typs 2 insbesondere freiwillig engagiert sind, d.h. auch ihren Sozialraum mitgestalten und an Betei- ligungsverfahren partizipieren. Jene, die dem Typ 2 zugeordnet wurden, engagieren sich nachberuflich in der Freiwilligenarbeit (z. B. in Wohn- und Pflegehäusern, in Stadtteilzentren, in Kirchen). Mitgestaltung findet hier einerseits im institutionellen Rahmen statt (z. B. Lernhilfe für Kinder; Begleit- und Besuchsdienste; politische und nachbarschaftliche Angebote). Margret (76 Jahre) sagt: „Na durch das, dass ich ja noch immer sehr politisch tätig bin, mache ich viele Wege, weil ich auch mit vielen Menschen $\mathrm{zu}$ tun hab und weil ich auch für meinen Bezirk noch immer Veranstaltungen organisiere. "Sozialräumliche Gestaltung hat aber auch einen „unsichtbaren“ Charakter: So zeigen aktive Ältere des Typs 1 und 2 auch, welche Grünflächen sie pflegen (z. B. kleine Grünflächen oder Baumscheiben) oder welche „Offenen Bücherschränke" sie im Wohnbezirk aufräumen, um einen Beitrag zur Verschönerung und Ordnung der unmittelbaren Erholungsräume zu leisten.

Bewohner können sich aktiv-gestaltend bis hin zu partizipativ-gestaltend einbringen, wenn Angebote auch tatsächlich mitgestaltet werden können (z.B. Mitgestaltung von Inhalten, Leitung und Wahl der Leitung, zeitliche Strukturen). Viele bestehende Angebote sind eher auf eine passive Teilnahme ausgerichtet. Auch diese Angebote sind wichtig, denn nicht allen älteren Menschen ist die Beteiligung an partizipativen Gestaltungsprozessen möglich.

\section{Fazit für die Praxis}

- Bisherige Studien aus dem Bereich der Gesundheitsförderung und der Stadteilarbeit fokussieren zu wenig auf den Sozialraum und wie dieser von Älteren genutzt und mitgestaltet wird.

- Die vorliegende Studie zeigt die Wichtigkeit in drei Kategorien auf: den Sozialraum wahrnehmen, ihn nutzen und aktiv bzw. partizipierend gestalten.

- In der partizipativen Gesundheitsförderung finden die Bewohner selbst als Experten ihrer Lebenswelt Anerkennung. Auch ihre sozialräumliche
Aneignung birgt wertvolle Erkenntnisse für die Planung.

- Die entwickelte Aktivitätstypologie differenziert Ältere nach ihrem Aktivitätspotential: Ältere, die bereits eine Verortung gefunden haben, können schwieriger aktiviert werden als jene, die freischwebend, d.h. auf der Suche sind. Die Freischwebenden haben für die Gesundheitsförderung das größte Aktivitätspotential.

- Die Nutzung der Typologie zu Aktivitätspotentialen Älterer kann Gesundheitsförderungsprozesse dabei unterstützen, ihre Zielgruppenadressierung zu verfeinern.

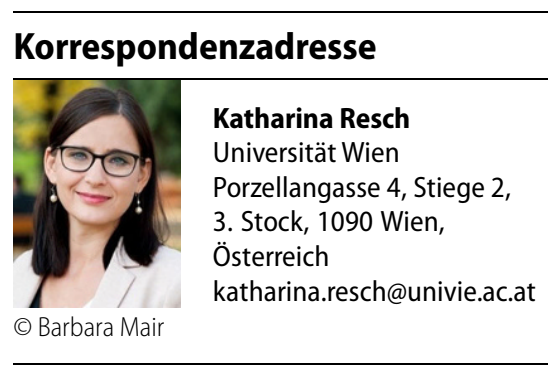

Danksagung. Unser Dank gilt den 30 älteren Personen, die an den Stadtteilbegehungen teilgenommen haben sowie dem Projektteam von "Gesund älter werden in Wien" (Wiener Gesundheitsförderung, Wiener Sozialdienste, Wiener HilfswerksNachbarschaftszentren, queraum.kultur- und sozialforschung, FH Campus Wien). Besonderer Dank gilt Simone Grandy und Ursula Hübel.

Förderung. Das Projekt wird zwischen 2017 und 2020 von der Wiener Gesundheitsförderung umgesetzt und aus Mitteln des Wiener Landesgesundheitsförderungsfonds finanziert. Der Beitrag stammt vom Erhebungsteam der Universität Wien im Rahmen des Projekts "Gesund älter werden in Wien".

Funding. Open access funding provided by University of Vienna.

\section{Einhaltung ethischer Richtlinien}

Interessenkonflikt. K. Resch, J. Demmer und A. FassI geben an, dass kein Interessenkonflikt besteht.

Alle beschriebenen Untersuchungen am Menschen wurden im Einklang mit nationalem Recht sowie gemäß der Deklaration von Helsinki von 1975 (in der aktuellen, überarbeiteten Fassung) durchgeführt. Von allen beteiligten älteren Personen liegt eine Einverständniserklärung vor. Dieser Beitrag beinhaltet keine Studien an Menschen oder Tieren.

Open Access Dieser Artikel wird unter der Creative Commons Namensnennung 4.0 International Lizenz (http://creativecommons.org/licenses/by/4.0/deed. de) veröffentlicht, welche die Nutzung, Vervielfäl- 


\section{Originalarbeit}

tigung, Bearbeitung, Verbreitung und Wiedergabe in jeglichem Medium und Format erlaubt, sofern Sie den/die ursprünglichen Autor(en) und die Quelle ordnungsgemäß nennen, einen Linkzur Creative Commons Lizenz beifügen und angeben, ob Änderungen vorgenommen wurden.

\section{Literatur}

1. Böhnisch L, Schröer W Soziale Räume im Lebenslauf. https://www.sozialraum.de/soziale-raeumeim-lebenslauf.php.Zugegriffen: 1. Aug. 2018

2. Büscher M, Urry J, Witchger K (2011) Mobile methods. Routledge, London, New York

3. Bundesministerium für Verkehr, Innovation und Technologie (2013) Mobilität im Alter. Ein Handbuch für PlanerInnen, EntscheidungsträgerInnen und Interessensvertreterlnnen. https:// www.bmvit.gv.at/service/publikationen/verkehr/ gesamtverkehr/downloads/mobilitaetalter_lang. pdf.Zugegriffen: 14. Dez. 2017

4. Deinet U (2018) Das Aneignungskonzept in der empirischen Praxis von Kindheits- und Jugendstudien. In: Deinet U, Reis C, Reutlinger C, Winkler M (Hrsg) Potentiale des Aneignungskonzepts. Beltz, Weinheim, Basel, S142-161

5. Deinet U (2014) Das Aneignungskonzept als Praxistheorie für die Soziale Arbeit. sozialraum.de. https://www.sozialraum.de/dasaneignungskonzept-als-praxistheorie-fuer-diesoziale-arbeit.php.Zugegriffen: 12.Dez. 2018

6. Deinet U, Reutlinger C (Hrsg) (2014) „Aneignung“ als Bildungskonzept derSozialpädagogik. Beiträge zur Pädagogik des Kindes und Jugendalters in Zeiten entgrenzter Lernorte. VS, Wiesbaden

7. Kessl F, Reutlinger C (2010) Sozialraum. Eine Einführung.VS, Wiesbaden

8. Kluge S (2000) Empirisch begründete Typenbildung in der qualitativen Sozialforschung. Forum Qualitative Sozialforschung (1). http://www. qualitative-research.net/index.php/fqs/article/ viewFile/1124/2498.Zugegriffen: 15. Okt. 2018

9. Krisch R (2003) Methoden einer sozialräumlichen Lebensweltanalyse. In:Deinet U, Krisch R(Hrsg) Der sozialräumliche Blick in der Jugendarbeit. Leske + Budrich, Opladen

10. Kusenbach M (2008) Mitgehen als Methode. Der "Go-Along" in der phänomenologischen Forschungspraxis. In: Dreher J, Pfadenhauer M, Raab J, Schnettler B, Stegmaier P (Hrsg) Phänomenologie und Soziologie. Theoretische Positionen, aktuelle Problemfelder und empirische Umsetzungen. VS, Wiesbaden, S349-358

11. Löw M, Steets S, Stoetzer S (2008) Einführung in die Stadt- und Raumsoziologie. Budrich, Opladen, Farmington Hills

12. Stadt Wien (Hrsg) (2017) Statistisches Jahrbuch der Stadt Wien 2017. MA 23 - Wirtschaft, Arbeit und Statistik. https://www.wien.gv.at/statistik/ publikationen/jahrbuch.html. Zugegriffen: 15. Okt.2018

13. Strauss A, Corbin J (1996) Grounded Theory: Grundlagen qualitativer Sozialforschung. Beltz, Weinheim

14. Wolter B (2011) Aneignung und Verlust des städtischen Raumes im Alter. In: Bergmann M, Lange B (Hrsg) Eigensinnige Geographien. Städtische Raumaneignungen als Ausdruck gesellschaftlicher Teilhabe. VS, Wiesbaden, S195-211

15. https://www.wig.or.at/Gesund\%20älter \%20werden\%20in\%20Wien.2109.0.html. Zugegriffen:13. Dezember 2018 